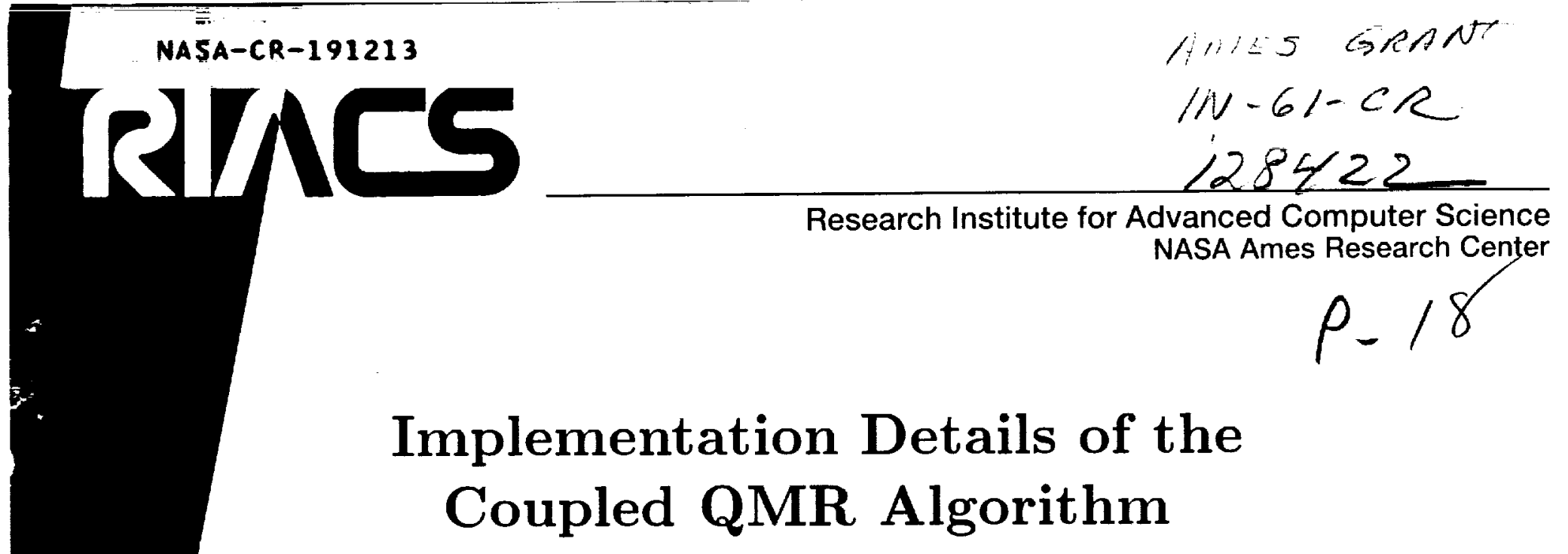

Roland W. Freund and Noël M. Nachtigal

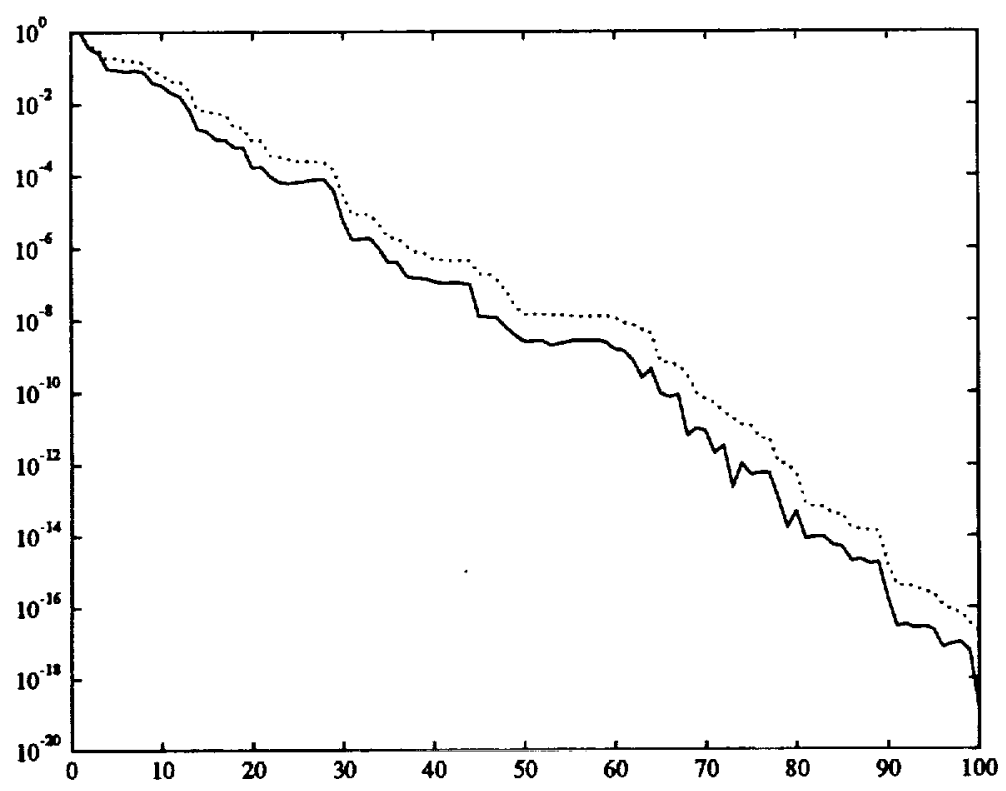

RIACS Technical Report 92.19

October 1992

(NASA-CR-191213) IMPLEMENTATION

N93-12301

DETAILS OF THE COUPLED QMR

ALGORITHM (Research Inst. for

Advanced Computer Science) $18 \mathrm{p}$

Unclas 



\title{
Implementation Details of the Coupled QMR Algorithm
}

\author{
Roland W. Freund and Noël M. Nachtigal
}

The Research Institute for Advanced Computer Science is operated by Universities Space Research Association (USRA),

The American City Building, Suite 311, Columbia, MD 21044, (301)730-2656.

Work reported herein was supported in part by DARPA via Cooperative Agreement NCC 2-387 between NASA and USRA. 
<smiles></smiles> 


\title{
Implementation details of the coupled QMR algorithm
}

\author{
Roland W. Freund and Noël M. Nachtigal
}

\begin{abstract}
The original quasi-minimal residual method (QMR) relies on the threeterm look-ahead Lanczos process to generate basis vectors for the underlying Krylov subspaces. However, empirical observations indicate that, in finite precision arithmetic, three-term vector recurrences are less robust than mathematically equivalent coupled twoterm recurrences. Therefore, we recently proposed a new implementation of the QMR method based on a coupled two-term look-ahead Lanczos procedure. In this paper, we describe implementation details of this coupled QMR algorithm, and we present results of numerical experiments.
\end{abstract}

\section{Introduction}

Recently, we proposed a new Krylov subspace iteration, the quasi-minimal residual algorithm (QMR) [5], for solving general nonsingular non-Hermitian systems of linear equations

$$
A x=b .
$$

The QMR method has two main ingredients: the look-ahead Lanczos process, and a quasi-minimal residual condition. The look-ahead Lanczos algorithm is used to generate-with low work and storage requirements-basis vectors for the underlying Krylov subspaces. Furthermore, look-ahead techniques are used to avoid possible breakdowns in the classical Lanczos algorithm [7], except for so-called incurable breakdowns. Once the Lanczos basis is constructed, the quasi-minimal residual property is used to select the QMR iterates from the Krylov subspaces. As was shown in [5], the QMR iterates are always well defined, and the quasi-minimal residual condition leads to a smooth and nearly monotone convergence behavior. In addition, thanks to the quasi-minimal residual property, it is possible to prove convergence results for the QMR algorithm. The result is a method with several desirable numerical and theoretical properties.

In the original QMR algorithm, the look-ahead Lanczos method used generates the basis vectors for the Krylov subspaces by means of three-term recurrences. It has been observed that, in finite precision arithmetic, vector iterations based on three-term recursions are usually less robust than mathematically equivalent 
coupled two-term vector recurrences. Therefore, in [6], we proposed a new implementation of the QMR algorithm, based on a coupled two-term recurrence formulation of the Lanczos algorithm. Together with the derivation, we discussed in [6] the properties of the new implementation, and presented numerical results showing that the new method is more robust than the original QMR algorithm. In this paper, we discuss in more detail the implementation of the new algorithm; in particular, we focus on the implementation of the coupled two-term version of the look-ahead Lanczos algorithm. We also give several new numerical examples.

The outline of the paper is as follows. In Section 2, we recall the three-term look-ahead Lanczos process that was proposed in [3]. Then, in Section 3, we review the coupled two-term look-ahead Lanczos algorithm that was proposed in [6], and in Section 4 we give implementation details of the new algorithm. In Section 5, we briefly recall how the QMR approach can be combined with the coupled Lanczos algorithm to obtain a new implementation of the QMR method, and in Section 6, we report results of numerical experiments with this new QMR algorithm. Finally, in Section 7 , we make some concluding remarks.

Throughout the paper, all vectors and matrices are allowed to have real or complex entries. As usual, $M^{T}=\left[m_{k j}\right]$ denotes the transpose of the matrix $M=\left[m_{j k}\right]$. We use $\sigma_{\min }(M)$ for the smallest singular value of $M$, while the vector norm $\|x\|:=\sqrt{x^{H} x}$ is always the Euclidean norm. We denote by

$$
K_{n}(c, B):=\operatorname{span}\left\{c, B c, \ldots, B^{n-1} c\right\}
$$

the $n$th Krylov subspace of $C^{N}$ generated by $c \in C^{N}$ and the $N \times N$ matrix $B$. Finally, it is always assumed that $A$ is an $N \times N$ matrix, singular or nonsingular.

\section{The three-term look-ahead Lanczos algorithm}

The Lanczos process is a method that builds basis vectors for two Krylov subspaces, with low work and storage requirements. Given two starting vectors, $v_{1}$ and $w_{1} \in C^{N}$, the algorithm computes two sequences of vectors, $\left\{v_{j}\right\}_{j=1}^{n}$ and $\left\{w_{j}\right\}_{j=1}^{n}$, such that, for $n=1,2, \ldots$,

$$
\begin{aligned}
\operatorname{span}\left\{v_{1}, v_{2}, \ldots, v_{n}\right\} & =K_{n}\left(v_{1}, A\right), \\
\operatorname{span}\left\{w_{1}, w_{2}, \ldots, w_{n}\right\} & =K_{n}\left(w_{1}, A^{T}\right) .
\end{aligned}
$$

In addition, the two sets of vectors are required to obey a biorthogonality relation. Ideally, one would like to impose the condition

$$
w_{n}^{T} v_{j}=w_{j}^{T} v_{n}=0 \quad \text { for all } j<n .
$$

This is done in the Lanczos process, as proposed by Lanczos in [7]. However, it turns out that it is not always possible or numerically stable to construct vectors satisfying $(2.2)$, as exact breakdowns $\left(w_{n}^{T} v_{n}=0\right)$ or near-breakdowns $\left(w_{n}^{T} v_{n}\right.$ is small in some sense) may arise. This poses a problem, since the construction 
of the pair $v_{n+1}$ and $w_{n+1}$ obeying (2.2) involves division by $w_{n}^{T} v_{n}$. As a remedy, one relaxes the biorthogonality condition, requiring instead that, in case of a breakdown, the relations (2.2) hold only for some range of $j$ up to, but not equal to, $n$. This leads to so-called look-ahead Lanczos algorithms, which skip over the exact and near-breakdowns. The original QMR algorithm is based on a look-ahead Lanczos method proposed by Freund, Gutknecht, and Nachtigal [3], which we briefly review next.

Like the classical algorithm, the look-ahead Lanczos algorithm [3] generates vectors $\left\{v_{j}\right\}_{j=1}^{n}$ and $\left\{w_{j}\right\}_{j=1}^{n}$ with (2.1). In addition, they satisfy the biorthogonality relation

$$
W_{n}^{T} V_{n}=D_{n}
$$

where $D_{n}$ is a block diagonal matrix whose structure is discussed below, and

$$
V_{n}:=\left[\begin{array}{llll}
v_{1} & v_{2} & \cdots & v_{n}
\end{array}\right] \text { and } W_{n}:=\left[\begin{array}{llll}
w_{1} & w_{2} & \cdots & w_{n}
\end{array}\right] \text {. }
$$

This means that some of the vectors $\left\{v_{j}\right\}_{j=1}^{n}$ and $\left\{w_{j}\right\}_{j=1}^{n}$ do in fact satisfy the full biorthogonality (2.2). These vectors are called regular, and they form a subsequence $\left\{v_{n_{j}}\right\}_{j=1}^{l}$ and $\left\{w_{n_{j}}\right\}_{j=1}^{l}$, where

$$
1=: n_{1}<n_{2}<\cdots<n_{l} \leq n<n_{l+1}, \quad l:=l(n) .
$$

All vectors that are not regular are called inner. The regular vectors are used to partition $\left\{v_{j}\right\}_{j=1}^{n}$ and $\left\{w_{j}\right\}_{j=1}^{n}$ into blocks. By convention, one defines blocks $V^{(j)}$, of size $N \times h_{j}$, containing the regular vector $v_{n_{j}}$ and all inner vectors-if any-between $v_{n_{j}}$ and $v_{n_{j+1}}$ :

$$
V^{(j)}=\left[\begin{array}{llll}
v_{n_{j}} & v_{n_{j}+1} & \cdots
\end{array}\right] .
$$

A look-ahead step is then defined in terms of building such a block. Hence the integer $l$ in (2.4) is just the number of look-ahead steps taken during the first $n$ steps of the Lanczos algorithm. Moreover, $h_{j}$ is called the length of the $j$ th lookahead step. The structure of the sequence $\left\{w_{j}\right\}_{j=1}^{n}$ parallels that of the sequence $\left\{v_{j}\right\}_{j=1}^{n}$, so that $V_{n}$ and $W_{n}$ can be written as

$$
V_{n}=\left[\begin{array}{llll}
V^{(1)} & V^{(2)} & \ldots & V^{(l)}
\end{array}\right] \text { and } W_{n}=\left[\begin{array}{lllll}
W^{(1)} & W^{(2)} & \cdots & W^{(l)}
\end{array}\right],
$$

and $D_{n}$ in (2.3) is given by

$$
D_{n}=\operatorname{diag}\left(D^{(1)}, D^{(2)}, \ldots, D^{(l)}\right), \quad D^{(j)}:=\left(W^{(j)}\right)^{T} V^{(j)} .
$$

The choice of whether to build a regular or an inner vector is determined at each step, based on the particular look-ahead strategy used. It should also be pointed out that, even though the look-ahead Lanczos algorithm can handle most breakdowns, there remains a class of breakdowns, the so-called incurable breakdowns, which cannot be cured by look-ahead techniques. However, incurable breakdowns occur only in very particular circumstances, and they do not pose a problem in practice. Finally, since the scaling of the Lanczos vectors is not determined by (2.1) and (2.3), the look-ahead algorithm scales the vectors to have unit length:

$$
\left\|v_{n}\right\|=\left\|w_{n}\right\|=1, \quad n=1,2, \ldots
$$


The main point of the look-ahead Lanczos process is that vectors satisfying (2.1) and (2.3) can be constructed by means of short block three-term recurrences. These recurrences can be written compactly as

$$
\begin{aligned}
A V_{n} & =V_{n+1} H_{n}, \\
A^{T} W_{n} & =W_{n+1} \Gamma_{n+1}^{-1} H_{n} \Gamma_{n},
\end{aligned}
$$

where $H_{n}$ is an $(n+1) \times n$ block tridiagonal matrix,

$$
\Gamma_{n}:=\operatorname{diag}\left(\gamma_{1}, \gamma_{2}, \ldots, \gamma_{n}\right), \quad \text { where } \gamma_{j}:= \begin{cases}1, & \text { if } j=1, \\ \gamma_{j-1} \rho_{j} / \xi_{j}, & \text { if } j>1,\end{cases}
$$

is a diagonal scaling matrix with positive diagonal entries, and $\rho_{j}$ and $\xi_{j}$ are scale factors used to ensure that $v_{j}$ and $w_{j}$, respectively, obey the scaling (2.5). Since the recurrences used to build $v_{n+1}$ and $w_{n+1}$ are short, the look-ahead algorithm has low work and storage requirements, making it an attractive method for building bases for Krylov subspaces. However, it has been observed that, in finite precision arithmetic, three-term vector recurrences are less robust than mathematically equivalent coupled two-term recurrences. This was our motivation in proposing in [6] a different implementation of the look-ahead Lanczos algorithm, based on coupled two-term recurrences. Next, we review this algorithm.

\section{The coupled two-term look-ahead Lanczos process}

The coupled two-term Lanczos process is an alternate way of generating the Lanczos basis vectors: The algorithm generates, in addition to the Lanczos vectors $\left\{v_{j}\right\}_{j=1}^{n}$ and $\left\{w_{j}\right\}_{j=1}^{n}$, a second set of basis vectors, $\left\{p_{j}\right\}_{j=1}^{n}$ and $\left\{q_{j}\right\}_{j=1}^{n}$, such that, for $n=1,2, \ldots$,

$$
\begin{aligned}
\operatorname{span}\left\{p_{1}, p_{2}, \ldots, p_{n}\right\} & =K_{n}\left(v_{1}, A\right), \\
\operatorname{span}\left\{q_{1}, q_{2}, \ldots, q_{n}\right\} & =K_{n}\left(w_{1}, A^{T}\right) .
\end{aligned}
$$

For simplicity, we will sometimes refer to the Lanczos vectors $\left\{v_{j}\right\}_{j=1}^{n}$ and $\left\{w_{j}\right\}_{j=1}^{n}$ as the $\mathrm{V}-\mathrm{W}$ sequence, and to the auxiliary vectors $\left\{p_{j}\right\}_{j=1}^{n}$ and $\left\{q_{j}\right\}_{j=1}^{n}$ as the $\mathrm{P}-\mathrm{Q}$ sequence. The four sets of basis vectors are generated using coupled two-term recurrences of the form:

$$
\begin{aligned}
V_{n} & =P_{n} U_{n}, & A P_{n} & =V_{n+1} L_{n}, \\
W_{n} & =Q_{n} \Gamma_{n}^{-1} U_{n} \Gamma_{n}, & A^{T} Q_{n} & =W_{n+1} \Gamma_{n+1}^{-1} L_{n} \Gamma_{n},
\end{aligned}
$$

Here,

$$
P_{n}:=\left[\begin{array}{llll}
p_{1} & p_{2} & \cdots & p_{n}
\end{array}\right] \text { ad } Q_{n}:=\left[\begin{array}{llll}
q_{1} & q_{2} & \cdots & q_{n}
\end{array}\right],
$$

*The discussion that follows will not cover all the details and will not justify all the statements made. For full details, we refer the reader to [6]. 
while $U_{n}$ is an upper triangular matrix and $L_{n}$ is an upper Hessenberg matrix, given by

$$
U_{n}:=\left[\begin{array}{cccc}
1 & u_{12} & \cdots & u_{1 n} \\
0 & 1 & \ddots & \vdots \\
\vdots & \ddots & \ddots & u_{n-1, n} \\
0 & \cdots & 0 & 1
\end{array}\right] \text { and } L_{n}:=\left[\begin{array}{cccc}
l_{11} & l_{12} & \cdots & l_{1 n} \\
\rho_{2} & l_{22} & & \vdots \\
0 & \rho_{3} & \ddots & \vdots \\
\vdots & \ddots & \ddots & l_{n n} \\
0 & \cdots & 0 & \rho_{n+1}
\end{array}\right]
$$

and $\Gamma_{n}$ is the diagonal matrix defined in (2.8). As was shown in [6], the matrices $L_{n}$ and $U_{n}$ define a factorization of the block tridiagonal Hessenberg matrix $H_{n}$ generated by the three-term look-ahead Lanczos algorithm,

$$
H_{n}=L_{n} U_{n} \text {. }
$$

In addition, it is possible to reduce $L_{n}$ and $U_{n}$ to block bidiagonal matrices, by constructing the basis vectors $p_{n}$ and $q_{n}$ so as to be block $A$-biorthogonal. Here, similar to the $\mathrm{V}-\mathrm{W}$ sequence, the vectors $p_{n}$ and $q_{n}$ are also constructed using look-ahead techniques. For example, we again have blocks $P^{(j)}$,

$$
P^{(j)}=\left[\begin{array}{llll}
p_{m_{j}} & p_{m_{j}+1} & \cdots
\end{array}\right],
$$

where $p_{m}$ is called regular, the other vectors in the block are called inner, and the indices $m_{j}$ satisfy

$$
1=: m_{1}<m_{2}<\cdots<m_{k}<n \leq m_{k+1}, \quad k:=k(n) .
$$

The regular vectors $p_{m}$, satisfy the $A$-biorthogonality condition

$$
q_{i}^{T} A p_{m_{j}}=0 \text { for all } i<m_{j},
$$

while the inner vectors satisfy only a relaxed version of this condition. Once again, the structure of $Q_{n}$ parallels that of $P_{n}$, and the $A$-biorthogonality of the two sets of basis vectors can be written as:

$$
Q_{n}^{T} A P_{n}=E_{n}=\operatorname{diag}\left(E^{(1)}, E^{(2)}, \ldots, E^{(k)}\right), \quad E^{(j)}:=\left(Q^{(j)}\right)^{T} A P^{(j)} .
$$

Before we consider the implementation details, let us briefly discuss an outline of the algorithm. At each iteration, the process consists of the following four basic steps.

Algorithm 3.1. (Overview of the coupled algorithm with look-ahead)

1) Decide whether to construct $p_{n}$ and $q_{n}$ as regular or inner vectors.

2) Compute $p_{n}$ and $q_{n}$ as either regular or inner vectors.

3) Decide whether to construct $v_{n+1}$ and $w_{n+1}$ as regular or inner vectors.

4) Compute $v_{n+1}$ and $w_{n+1}$ as either regular or inner vectors. 
Steps 1) and 3) are the basis of the look-ahead strategy, and they each consist of three checks. Recall from (2.6) and (2.7) that the Lanczos vectors $v_{n+1}$ and $w_{n+1}$ can be obtained from the previous Lanczos vectors by a block three-term recurrence. Similarly, it is possible to show that the vectors $p_{n}$ and $q_{n}$ also have a block three-term recurrence, of the form

$$
A P_{n-1}=P_{n} G_{n-1} \text { and } A^{T} Q_{n-1}=Q_{n} \Gamma_{n}^{-1} G_{n-1} \Gamma_{n-1}
$$

where

$$
G_{n-1}:=U_{n} L_{n-1} .
$$

The look-ahead strategy for the two pairs of sequences is then similar, and was first proposed in [3]. In Step 1), the algorithm checks whether:

$$
\begin{aligned}
& n(A)\left\|p_{n}\right\| \geq \sum_{i=m_{k-1}}^{n-1}\left|\left(U_{n} L_{n-1}\right)_{i, n-1}\right|\left\|p_{i}\right\|, \\
& n(A)\left\|q_{n}\right\| \geq \sum_{i=m_{k-1}}^{\sigma_{\min }\left(E^{(k)}\right) \geq e p s,} \frac{\gamma_{n-1}}{\gamma_{i}}\left|\left(U_{n} L_{n-1}\right)_{i, n-1}\right||| q_{i} \| .
\end{aligned}
$$

Here, eps is machine epsilon, and $n(A)$ is an estimate for the norm of $A$. The vectors $p_{n}$ and $q_{n}$ are built as regular vectors only if all three of the above checks are satisfied. Likewise, in Step 3), the algorithm checks whether:

$$
\begin{gathered}
\sigma_{\min }\left(D^{(l)}\right) \geq \text { eps } \\
n(A) \geq \sum_{i=n_{1-1}}^{n}\left|\left(L_{n} U_{n}\right)_{i n}\right|, \\
n(A) \geq \sum_{i=n_{i-1}}^{n} \frac{\gamma_{n}}{\gamma_{i}}\left|\left(L_{n} U_{n}\right)_{i n}\right|,
\end{gathered}
$$

and again, the vectors $v_{n+1}$ and $w_{n+1}$ are built as regular vectors only if all three of the checks are passed. The motivation for these checks can be found in $[3,6]$. Here, we will only note that the look-ahead strategy proposed will build regular vectors in preference to inner vectors, and thus it will take as few look-ahead steps as possible.

Once the decisions in Steps 1) and 3) are taken, the next vectors $p_{n}$ and $q_{n}$, and $v_{n+1}$ and $w_{n+1}$, are built in Steps 2) and 4). For $p_{n}$ and $q_{n}$, let $k^{\star}$ denote the number of the row of the first possible nonzero element in the $n$th column of $U_{n}$. It can be shown that

$$
k^{\star}=\max \left\{j \mid 1 \leq j \leq k \text { and } m_{j} \leq \max \left\{1, n_{l}-1\right\}\right\} .
$$

From (3.3), both the regular and the inner vectors have the same coefficients $U_{m_{k}: m_{k}-1, n}$, given by ${ }^{\dagger}$

$$
\begin{aligned}
U_{m_{k}: m_{k}-1, n}=\left(\operatorname { d i a g } \left(E^{\left(k^{*}\right)},\right.\right. & \left.\left.E^{\left(k^{*}+1\right)}, \ldots, E^{(k-1)}\right)\right)^{-1} \\
& \cdot\left[\begin{array}{llll}
Q^{\left(k^{*}\right)} & Q^{\left(k^{*}+1\right)} & \cdots & Q^{(k-1)}
\end{array}\right]^{T} A v_{n} .
\end{aligned}
$$

tWe denote $x_{i: j}=\left[\begin{array}{llll}x_{i} & x_{i+1} & \cdots & x_{j}\end{array}\right]^{T}$. 
For the regular vectors, the coefficients $U_{m_{k}: n-1, n}$ are determined by the condition (3.2),

$$
U_{m_{k}: n-1, n}=\left(E^{(k)}\right)^{-1}\left(Q^{(k)}\right)^{T} A v_{n},
$$

while for the inner vectors, the coefficients $U_{m_{k}: n-1, n}$ are arbitrary:

$$
u_{i n}=\zeta_{\text {in }} \in \mathbb{C}, \text { for } i=m_{k}, m_{k}+1, \ldots, n-1 .
$$

This completes the computation of the recurrence coefficients for $p_{n}$ and $q_{n}$, and the vectors are then computed from

$$
\begin{aligned}
& p_{n}=v_{n}-\sum_{i=m_{k^{\star}}}^{n-1} p_{i} u_{i n}, \\
& q_{n}=w_{n}-\sum_{i=m_{k^{\star}}}^{n-1} q_{i} u_{i n}\left(\gamma_{n} / \gamma_{i}\right) .
\end{aligned}
$$

For $v_{n+1}$ and $w_{n+1}$, let $l^{\star}$ denote the number of the row of the first possible nonzero element in the $n$th column of $L_{n}$. It can be shown that

$$
r^{\star}=\max \left\{j \mid 1 \leq j \leq l \text { and } n_{j} \leq m_{k}\right\} .
$$

Again, by (2.3), both the regular and the inner vectors have the same coefficients $L_{n_{1}: n_{1}-1, n}$, given by

$$
\begin{aligned}
L_{n_{1}: n_{l}-1, n}=\left(\operatorname { d i a g } \left(D^{\left(l^{*}\right)},\right.\right. & \left.\left.D^{\left(l^{*}+1\right)}, \ldots, D^{(l-1)}\right)\right)^{-1} \\
\cdot & {\left[\begin{array}{llll}
W^{\left(l^{*}\right)} & W^{\left(l^{\star}+1\right)} & \ldots & W^{(l-1)}
\end{array}\right]^{T} A p_{n} . }
\end{aligned}
$$

For the regular vectors, the coefficients $L_{n_{1}: n, n}$ are determined by the condition (2.2),

$$
L_{n_{l}: n, n}=\left(D^{(l)}\right)^{-1}\left(W^{(l)}\right)^{T} A p_{n}
$$

while for the inner vectors, the coefficients $L_{n !: n, n}$ are arbitrary:

$$
l_{\text {in }}=\eta_{\text {in }} \in \mathrm{C} \text { for } i=n_{l}, n_{l}+1, \ldots, n \text {. }
$$

Once the recurrence coefficients for $v_{n+1}$ and $w_{n+1}$ are computed, the vectors are constructed by scaling the vectors

$$
\begin{aligned}
& \tilde{v}_{n+1}=A p_{n}-\sum_{i=n_{1^{\star}}}^{n} v_{i} l_{i n}, \\
& \tilde{w}_{n+1}=A^{T} q_{n}-\sum_{i=n_{1^{\star}}}^{n} w_{i} l_{i n}\left(\gamma_{n} / \gamma_{i}\right),
\end{aligned}
$$

to have unit length. 


\section{Implementation details}

We now turn to a detailed description of the implementation of Algorithm 3.1. If the coupled two-term Lanczos process is run without any look-ahead steps, it will require two inner products at each step in order to compute all the coefficients of the recurrence formulas. Hence, the goal for the look-ahead implementation is to also require only two inner products per step for all the recurrence coefficients. Recall that the look-ahead strategy (3.6) and (3.7) for the $P-Q$ sequence and the normalization (2.5) require a total of four norm computations, so that the implementation will require two inner products and four norms per iteration.

To begin, let us introduce the auxiliary matrices

$$
F_{n}:=W_{n}^{T} A P_{n} \quad \text { and } \quad \tilde{F}_{n}:=Q_{n}^{T} A V_{n} \text {, }
$$

whose columns are needed in (3.14) and in (3.11). In addition, we will make use of the following symmetry relations from [6]:

$$
\begin{aligned}
& D_{n} \Gamma_{n}=\left(D_{n} \Gamma_{n}\right)^{T}, \\
& E_{n} \Gamma_{n}=\left(E_{n} \Gamma_{n}\right)^{T}, \\
& F_{n} \Gamma_{n}=\left(\tilde{F}_{n} \Gamma_{n}\right)^{T} .
\end{aligned}
$$

The $n$th iteration of the implementation will update the matrices $D_{n-1}, E_{n-1}$, $F_{n-1}, L_{n-1}, U_{n-1}, P_{n-1}, Q_{n-1}, V_{n}$, and $W_{n}$, to $D_{n}, E_{n}, F_{n}, L_{n}, U_{n}, P_{n}, Q_{n}$, $V_{n+1}$, and $W_{n+1}$, respectively. We first list an outline of the algorithm as we have implemented it.

Algorithm 4.1. (Coupled algorithm with look-ahead)

0) Choose $v_{1}, w_{1} \in \mathrm{C}^{N}$ with $\left\|v_{1}\right\|=\left\|w_{1}\right\|=1$, and compute $w_{1}^{T} v_{1}$.

Set $k=1, m_{k}=1, l=1, n_{l}=1$.

For $n=1,2, \ldots$, do:

1) Update $D_{n-1}$ to $D_{n}$.

2) Determine $k^{\star}$ from $(3.10)$ :

$$
k^{\star}=\max \left\{j \mid 1 \leq j \leq k \text { and } m_{j} \leq \max \left\{1, n_{l}-1\right\}\right\} .
$$

3) Compute $F_{n, 1: n-1}$ from (4.4) below, using $L_{n-1}$ and $D_{n, 1: n}$.

Then compute $\tilde{F}_{1: n-1, n}$ from (4.3).

4) Check whether $E^{(k)}$ is nonsingular:

$$
\text { innerp }=\sigma_{\min }\left(E^{(k)}\right)<\text { eps. }
$$

5) Compute the part of $U_{1: n, n}$ that is determined by biorthogonality:

$$
\begin{aligned}
U_{m_{1}: m_{1+1}-1, n} & =\left(E^{(i)}\right)^{-1}\left(Q^{(i)}\right)^{T} A v_{n} \\
& =\left(E^{(i)}\right)^{-1} \tilde{F}_{m_{i}: m_{1+1}-1, n}, \quad i=k^{\star}, \ldots, k-1 .
\end{aligned}
$$

If innerp, go to 6). Otherwise, set

$$
U_{m_{k}: n-1, n}=\left(E^{(k)}\right)^{-1}\left(Q^{(k)}\right)^{T} A v_{n}=\left(E^{(k)}\right)^{-1} \tilde{F}_{m_{k}: n-1, n} .
$$


6) Build the part of $p_{n}$ and $q_{n}$ that is common to both regular and inner vectors:

$$
\begin{aligned}
& p_{n}=v_{n}-\sum_{i=m_{k^{*}}}^{m_{k}-1} p_{i} u_{i n}, \\
& q_{n}=w_{n}-\sum_{i=m_{k^{*}}}^{m_{k}-1} q_{i} u_{i n}\left(\gamma_{n} / \gamma_{i}\right) .
\end{aligned}
$$

If innerp, go to 11).

7) Build $G_{m_{k}: n-1, n-1}$ and check the coefficient $G_{m_{k-1}: n-1, n-1}$. If innerp, go to 11).

8) Build $p_{n}$ and $q_{n}$ as regular vectors:

$$
\begin{aligned}
& p_{n}=p_{n}-\sum_{i=m_{k}}^{n-1} p_{i} u_{i n} \\
& q_{n}=q_{n}-\sum_{i=m_{k}}^{n-1} q_{i} u_{i n}\left(\gamma_{n} / \gamma_{i}\right)
\end{aligned}
$$

Compute $A p_{n}, q_{n}^{T} A p_{n},\left\|p_{n}\right\|$, and $\left\|q_{n}\right\|$.

9) Build and check the coefficient $G_{m_{k}: n-1, n}$. If innerp, go to 11).

10) Set $m_{k+1}=n, k=k+1$, and go to 12).

11) Choose the inner recurrence coefficients $u_{i n}, i=m_{k}, \ldots, n-1$, and build $p_{n}$ and $q_{n}$ as inner vectors:

$$
\begin{aligned}
& p_{n}=p_{n}-\sum_{i=m_{k}}^{n-1} p_{i} u_{i n}, \\
& q_{n}=q_{n}-\sum_{i=m_{k}}^{n-1} q_{i} u_{i n}\left(\gamma_{n} / \gamma_{i}\right) .
\end{aligned}
$$

Compute $A p_{n}, q_{n}^{T} A p_{n},\left\|p_{n}\right\|$, and $\left\|q_{n}\right\|$.

12) If $\left\|p_{n}\right\|=0$, or $\left\|q_{n}\right\|=0$, then stop.

13) Compute $A^{T} q_{n}$.

14) Update $E_{n-1}$ to $E_{n}$.

15) Determine ${ }^{\star}$ from (3.13):

$$
l^{\star}=\max \left\{j \mid 1 \leq j \leq l \text { and } n_{j} \leq m_{k}\right\} .
$$

16) Compute $F_{1: n, n}$ from (4.5) below, using $E_{n}$ and $U_{n}$.

17) Check whether $D^{(l)}$ is nonsingular:

$$
\text { innerv }=\sigma_{\min }\left(D^{(l)}\right)<\text { eps. }
$$


18) Compute the part of $L_{1: n, n}$ that is determined by biorthogonality:

$$
\begin{aligned}
L_{n_{i}: n_{i+1}-1, n} & =\left(D^{(i)}\right)^{-1}\left(W^{(i)}\right)^{T} A p_{n} \\
& =\left(D^{(i)}\right)^{-1} F_{n_{i}: n_{i+1}-1, n}, \quad i=l^{\star}, \ldots, l-1 .
\end{aligned}
$$

If innerv, go to 19). Otherwise, set

$$
L_{n ! n, n}=\left(D^{(l)}\right)^{-1}\left(W^{(l)}\right)^{T} A p_{n}=\left(D^{(l)}\right)^{-1} F_{n_{1}: n, n} .
$$

19) Build the part of $v_{n+1}$ and $w_{n+1}$ that is common to both regular and inner vectors:

$$
\begin{aligned}
& \tilde{v}_{n+1}=A p_{n}-\sum_{i=n_{1 *}}^{n_{1}-1} v_{i} l_{i n}, \\
& \tilde{w}_{n+1}=A^{T} q_{n}-\sum_{i=n_{1 *}}^{n_{1}-1} w_{i} l_{i n}\left(\gamma_{n} / \gamma_{i}\right)
\end{aligned}
$$

If innerv, go to 24).

20) Build $H_{n_{l}: n, n}$ and check the coefficient $H_{n_{1-1}: n, n}$.

If innerv, go to 24).

21) Build $v_{n+1}$ and $w_{n+1}$ as regular vectors:

$$
\begin{aligned}
& \tilde{v}_{n+1}=\tilde{v}_{n+1}-\sum_{i=n_{l}}^{n} v_{i} l_{i n}, \\
& \tilde{w}_{n+1}=\tilde{w}_{n+1}-\sum_{i=n_{1}}^{n} w_{i} l_{i n}\left(\gamma_{n} / \gamma_{i}\right) .
\end{aligned}
$$

Compute $\rho_{n+1}=l_{n+1, n}=\left\|\tilde{v}_{n+1}\right\|, \xi_{n+1}=\left\|\tilde{w}_{n+1}\right\|$.

If $\rho_{n+1}=0$ or $\xi_{n+1}=0$, then stop.

Otherwise, set $\gamma_{n+1}=\gamma_{n} \rho_{n+1} / \xi_{n+1}$, and compute $\tilde{w}_{n+1}^{T} \tilde{v}_{n+1}$.

22) Build and check the coefficient $H_{n ! n, n+1}$. If innerv, go to 24).

23) Set $n_{l+1}=n+1, l=l+1$, and go to 25).

24) Choose the inner recurrence coefficients $l_{\text {in }}, i=n_{l}, \ldots, n$, and build $v_{n+1}$ and $w_{n+1}$ as inner vectors:

$$
\begin{aligned}
& \tilde{v}_{n+1}=\tilde{v}_{n+1}-\sum_{i=n_{l}}^{n} v_{i} l_{i n}, \\
& \tilde{w}_{n+1}=\tilde{w}_{n+1}-\sum_{i=n_{l}}^{n} w_{i} l_{i n}\left(\gamma_{n} / \gamma_{i}\right) .
\end{aligned}
$$

Compute $\rho_{n+1}=l_{n+1, n}=\left\|\tilde{v}_{n+1}\right\|, \xi_{n+1}=\left\|\tilde{w}_{n+1}\right\|$. 
If $\rho_{n+1}=0$ or $\xi_{n+1}=0$, then stop.

Otherwise, set $\gamma_{n+1}=\gamma_{n} \rho_{n+1} / \xi_{n+1}$, and compute $\tilde{w}_{n+1}^{T} \tilde{v}_{n+1}$.

25) Set

$$
\begin{gathered}
v_{n+1}=\tilde{v}_{n+1} / \rho_{n+1}, \quad w_{n+1}=\tilde{w}_{n+1} / \xi_{n+1} \\
w_{n+1}^{T} v_{n+1}=\tilde{w}_{n+1}^{T} \tilde{v}_{n+1} /\left(\rho_{n+1} \xi_{n+1}\right)
\end{gathered}
$$

Step 1. The diagonal term $w_{n}^{T} v_{n}$ has already been computed directly, at the end of the previous step. Next, using

$$
\begin{aligned}
F_{n-1} & =W_{n-1}^{T} A P_{n-1}=W_{n-1}^{T} V_{n} L_{n-1} \\
& =D_{n-1} L_{1: n-1,1: n-1}+l_{n, n-1} D_{1: n-1, n}\left[\begin{array}{llll}
0 & \cdots & 0 & 1
\end{array}\right]
\end{aligned}
$$

the remainder of the last column of $D_{n}$ is computed from $D_{n-1}, F_{n-1}$, and $L_{n-1}$. The last row of $D_{n}$ is obtained by symmetry, using (4.1).

Step 7. We build $G_{m_{k}: n-1, n-1}$, which would be the coefficient of the $P^{(k)}$ and $Q^{(k)}$ blocks in the three-term recurrences (3.4) for $p_{n}$ and $q_{n}$. Using (3.5), one has

$$
G_{i, n-1}=\sum_{j=i}^{n} u_{i j} l_{j, n-1}, \quad i=m_{k}, \ldots, n-1 .
$$

The coefficient $G_{m_{k-1}: m_{k}-1, n-1}$ has already been built as part of Step 9 ) at the previous iteration. We then check (3.6)-(3.7), and set innerp to TRUE if at least one of the two checks fails.

Step 9. We build $G_{m_{k}: n-1, n}$, which would be the coefficient of the $P^{(k)}$ and $Q^{(k)}$ blocks in the three-term recurrences (3.4) for $p_{n+1}$ and $q_{n+1}$. It is straightforward to show that

$$
G_{m_{k}: n-1, n}=\left(E^{(k)}\right)^{-1}\left(Q^{(k)}\right)^{T} A A p_{n} .
$$

Moreover, we have

$$
\begin{aligned}
Q_{n-1}^{T} A A p_{n} & =\left(A^{T} Q_{n-1}\right)^{T} A p_{n}=\left(Q_{n} \Gamma_{n}^{-1} U_{n} L_{n-1} \Gamma_{n-1}\right)^{T} A p_{n} \\
& =\Gamma_{n-1} L_{n-1}^{T} U_{n}^{T} \Gamma_{n}^{-1} Q_{n}^{T} A p_{n} \\
& =\Gamma_{n-1} L_{n-1}^{T} U_{n}^{T} \Gamma_{n}^{-1}\left[\begin{array}{llll}
0 & \cdots & 0 & q_{n}^{T} A p_{n}
\end{array}\right]^{T} \\
& =\frac{\gamma_{n-1}}{\gamma_{n}} l_{n, n-1}\left[\begin{array}{llll}
0 & \cdots & 0 & q_{n}^{T} A p_{n}
\end{array}\right]^{T} .
\end{aligned}
$$

We then check a subset of (3.6)-(3.7), and set innerp to TRUE if at least one of the two checks fails.

Step 14. The diagonal term $q_{n}^{T} A p_{n}$ has already been computed directly, as part of Step 8) or Step 11). Next, using

$$
F_{n}=W_{n}^{T} A P_{n}=\Gamma_{n} U_{n}^{T} \Gamma_{n}^{-1} Q_{n}^{T} A P_{n},
$$

the remainder of the last row of $E_{n}$ is computed from $E_{n-1}, F_{1: n, 1: n-1}$, and $U_{n}$. The last column of $E_{n}$ is obtained by symmetry, using (4.2). 
Step 20. We build $H_{n ! n, n}$, which would be the coefficient of the $V^{(l)}$ and $W^{(l)}$ blocks in the three-term recurrences (2.6) and (2.7) for $v_{n+1}$ and $w_{n+1}$. Using (3.1), one has

$$
H_{i n}=\sum_{j=i}^{n} l_{i j} u_{j n}, \quad i=n_{l}, \ldots, n
$$

The coefficient $H_{n_{1-1}: n_{1}-1, n}$ has already been built as part of Step 22) at the previous iteration. We then check (3.8)-(3.9), and set innerv to TRUE if at least one of the two checks fails.

Step 22. We build $H_{n ! n, n+1}$, which would be the coefficient of the $V^{(l)}$ and $W^{(l)}$ blocks in the three-term recurrences $(2.6)$ and $(2.7)$ for $v_{n+2}$ and $w_{n+2}$. It is straightforward to show that

$$
H_{n ! n, n+1}=\left(D^{(l)}\right)^{-1}\left(W^{(l)}\right)^{T} A v_{n+1} .
$$

Moreover, we have

$$
\begin{aligned}
W_{n}^{T} A v_{n+1} & =\left(A^{T} W_{n}\right)^{T} A v_{n+1}=\left(W_{n+1} \Gamma_{n+1}^{-1} L_{n} U_{n} \Gamma_{n}\right)^{T} v_{n+1} \\
& =\Gamma_{n} U_{n}^{T} L_{n}^{T} \Gamma_{n+1}^{-1} W_{n+1}^{T} v_{n+1} \\
& =\Gamma_{n} U_{n}^{T} L_{n}^{T} \Gamma_{n+1}^{-1}\left[\begin{array}{llll}
0 & \cdots & 0 & w_{n+1}^{T} v_{n+1}
\end{array}\right]^{T} \\
& =\frac{\gamma_{n}}{\gamma_{n+1}} l_{n+1, n}\left[\begin{array}{llll}
0 & \cdots & 0 & w_{n+1}^{T} v_{n+1}
\end{array}\right]^{T} .
\end{aligned}
$$

We then check a subset of (3.8)-(3.9), and set innerv to TRUE if at least one of the two checks fails.

We remark that the checks in steps 9) and 22) are actually slightly relaxed versions of (3.8)-(3.9), and (3.6)-(3.7), respectively, since the indices checked are only a subset of the full range appearing in (3.8)-(3.9) and (3.6)-(3.7). We also note that the algorithm above requires minimal inputs from the user. Recall that eps in steps 4) and 17) is machine epsilon. Furthermore, the estimate $n(A)$ for the norm of the matrix can be updated dynamically, as was done in [3].

The coupled Lanczos Algorithm 4.1 requires per iteration the computation of two inner products and four vector norms. We conclude this section by noting that, in Algorithm 4.1, the choice of the inner recurrence coefficients (3.12) and (3.15) is arbitrary. In our implementation of the algorithm, we have used

$$
\begin{aligned}
u_{n-1, n} & =1, \\
u_{n-2, n} & =1, \quad \text { when } \quad m_{k} \leq n-2, \\
u_{i n} & =0, \quad \text { for } \quad i=m_{k}, \ldots, n-3 \\
l_{n n} & =1, \\
l_{n-1, n} & =1, \quad \text { when } \quad n_{1} \leq n-1, \\
l_{i n} & =0, \quad \text { for } \quad i=n_{l}, \ldots, n-2,
\end{aligned}
$$

for the inner vector recurrence coefficients. 


\section{The coupled two-term QMR algorithm}

We now consider the quasi-minimal residual approach and briefly outline how it can be combined with the coupled two-term look-ahead Lanczos algorithm of Section 3 to obtain a new implementation of the QMR method. We note that the QMR algorithm was proposed in [5] for the case of nonsingular linear systems (1.1). It was later shown by Freund and Hochbruck [4] that the algorithm can also be applied to singular systems, and that it always generates well-defined iterates. However, these iterates converge to a meaningful solution only for the special case of consistent systems with coefficient matrices $A$ of index 1 . Here, we consider the QMR method for the general case of $N \times N$ linear systems, with singular or nonsingular coefficient matrices.

The QMR algorithm belongs to the family of Krylov subspace methods. Let $x_{0} \in C^{N}$ be an initial guess for the solution of (1.1), and $r_{0}=b-A x_{0}$ the corresponding initial residual, of length $\rho_{1}=\left\|r_{0}\right\|$. Choosing $v_{1}=r_{0} / \rho_{1}$ as the starting right Lanczos vector for Algorithm 4.1, and $w_{1}$ with $\left\|w_{1}\right\|=1$ as an arbitrary starting left Lanczos vector, one obtains the four basis sets, $V_{n}, W_{n}, P_{n}$, and $Q_{n}$, of which the ones of interest are $V_{n}$ and $P_{n}$, related by:

$$
V_{n}=P_{n} R_{n} \text { and } A P_{n}=V_{n+1} L_{n} .
$$

Once the basis vectors are constructed, the $n$th QMR iterate is selected from the shifted Krylov subspace $x_{0}+K_{n}\left(r_{0}, A\right)$ as

$$
x_{n}=x_{0}+P_{n} y_{n},
$$

where $y_{n} \in C^{n}$ is defined by the quasi-minimal residual condition

$$
\left\|f_{n+1}-L_{n} y_{n}\right\|=\min _{y \in C^{n}}\left\|f_{n+1}-L_{n} y\right\| .
$$

This is an $(n+1) \times n$ least-squares problem, where

$$
f_{n+1}:=\rho_{1} \cdot\left[\begin{array}{llll}
1 & 0 & \cdots & 0
\end{array}\right]^{T} \in \mathbf{R}^{n+1},
$$

and we have used the normalization (2.5) of the Lanczos vectors; otherwise, the least-squares problem above also involves a diagonal scaling matrix.

Note that, by setting

$$
z_{n}=\left(R_{n}\right)^{-1} y_{n}
$$

and inserting in (5.2), one obtains the equivalent least-squares problem

$$
\left\|f_{n+1}-L_{n} R_{n} z_{n}\right\|=\min _{z \in \mathcal{C}^{n}}\left\|f_{n+1}-L_{n} R_{n} z\right\|,
$$

which is exactly the least-squares problem solved by the QMR algorithm based on the three-term Lanczos process. Thus, the QMR iterates (5.1) are, in exact arithmetic, identical to the iterates of the original QMR algorithm [5]. However, as was shown in [6], in finite precision arithmetic, the coupled QMR algorithm is more robust than the three-term recurrence version.

Like the original QMR algorithm, the new implementation has a number of desirable properties. Since they are equivalent, all the theoretical properties of the 
three-term recurrence QMR algorithm carry over to the coupled version. The new method also shows a smooth and nearly monotone convergence curve. At all times during the iteration, an upper bound for the QMR residual norm is available at no extra cost, and, as the examples will show, this upper bound is a very good indicator of the convergence of the method. As in the original version, the QMR iterate has a short update formula, involving only one direction vector that can be updated with a two-term recurrence. This is slightly cheaper than in the old method, where the corresponding search directions for the update of the iterates had a three-term recurrence. Finally, the new version seems to be significantly more robust than the old version, though no theoretical results are available to explain the differences. For a full discussion of the properties of the two QMR algorithms, see $[5,6]$.

\section{Numerical experiments}

In this section, we present a few numerical examples with the new implementation of the QMR algorithm. All these examples were run either on a Cray-2 at the NASA Ames Research Center or on a Cray Y-MP at AT\&T Bell Laboratories, with a machine epsilon of about 5.0E-29.

In the plots below, we always show two curves, the computed scaled residual norm $\left\|r_{n}\right\| /\left\|r_{0}\right\|$ (solid line) and the residual norm upper bound (dotted line). Recall that the upper bound is available at each step at no extra cost.

Example 6.1. This example is taken from [2]. Here we consider the differential equation

$$
L u=f \quad \text { on }(0,1) \times(0,1) \times(0,1)
$$

where

$$
\begin{aligned}
L u:= & -\frac{\partial}{\partial x}\left(e^{-x y} \frac{\partial u}{\partial x}\right)-\frac{\partial}{\partial y}\left(e^{x y} \frac{\partial u}{\partial y}\right)-\frac{\partial}{\partial z}\left(e^{x y} \frac{\partial u}{\partial z}\right) \\
& +50(x+y+z) \frac{\partial u}{\partial x}+\left(\frac{1}{1+x+y+z}-250\right) u,
\end{aligned}
$$

with Dirichlet boundary conditions $u=0$. The right-hand side $f$ was chosen so that

$$
u=(1-x)(1-y)(1-z)\left(1-e^{-x}\right)\left(1-e^{-y}\right)\left(1-e^{-z}\right)
$$

is the exact solution of (6.1). We discretized (6.1) using centered differences on a $40 \times 40 \times 40$ grid with mesh size $h=1 / 41$. This leads to a linear system of order $N=64000$ with 438400 nonzero entries. The starting vector $w_{1}$ was a random vector, and the initial guess $x_{0}$ was zero. The example was run with a right SSOR preconditioner [1], with $\omega=1.0$. The algorithm stagnated at $1.6 \mathrm{E}-25$ after 119 steps. For the V-W sequence, it built 4 blocks of size 2 , and forced a block closure once. For the $\mathrm{P}-\mathrm{Q}$ sequence, it built 2 blocks of size 2 , and forced a block closure once. 


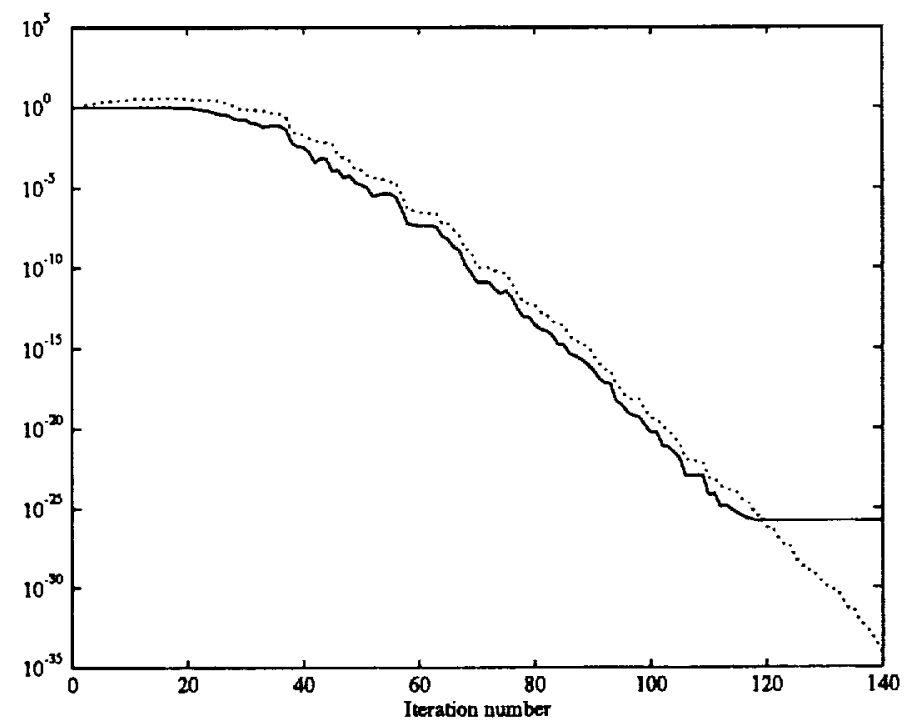

Figure 1: Convergence curves for Example 6.1.

Example 6.2. This example was provided to us by V. Venkatakrishnan [10], from the Numerical Aerodynamic Simulation Group at the NASA Ames Research Center. It comes from an unstructured 2-D Euler solver, and it corresponds to the system at the beginning of time-stepping. The linear system is of order $N=62424$ with 1717792 nonzero elements. The right-hand side $b$ and the starting vector $w_{1}$ were both random vectors, while the initial guess $x_{0}$ was zero. Once again, the example was run with a right SSOR preconditioner, with $\omega=1.0$. The algorithm was stopped once it reached $1.0 \mathrm{E}-20$, after 107 steps. For the $\mathrm{V}-\mathrm{W}$ sequence, it built 3 blocks of size 2 , and 1 block of size 3 . For the $P-Q$ sequence, it built no blocks, but forced a block closure once.

Example 6.3. This example was taken from [8], where McQuain and his collaborators investigated the applicability of iterative methods to linear systems arising in circuit simulation. In particular, they studied the solution of systems involving the Jacobians that arise when a homotopy algorithm is applied to the computation of the DC operating point of a circuit. While these linear systems seem to be rather difficult, they are not intractable. In the example, a Jacobian of order $N=1853$ with 8994 nonzero elements was considered; it is the first Jacobian from the IS7B sequence discussed in Section 5.3 of [8]. The right-hand side $b$ was obtained by moving the last column of the original rectangular $n \times(n+1)$ Jacobian to the other side. The starting guess $x_{0}$ was zero, and the starting vector $w_{1}$ was set $w_{1}=v_{1}=b$. The example was run with the variant described in [5] of Saad's ILUT preconditioner [9], with no additional fill-in allowed and a drop tolerance of 


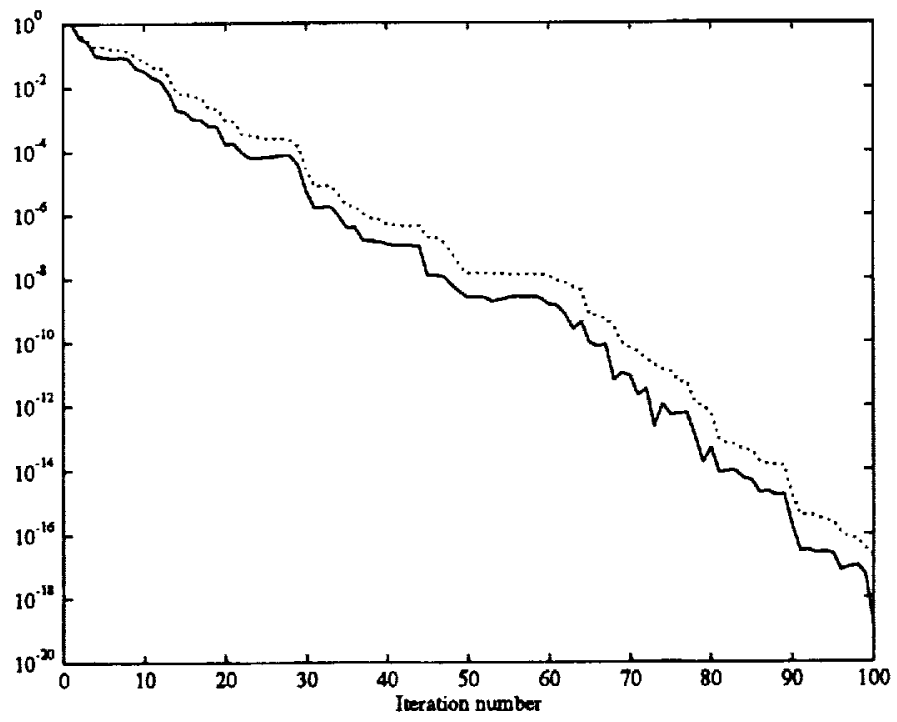

Figure 2: Convergence curves for Example 6.2.

0.001 , which generated a matrix $L$ with 4766 nonzero elements, and a matrix $U$ with 5034 nonzero elements. The algorithm stagnated at $8.7 \mathrm{E}-23$ after 67 steps. It built no look-ahead blocks.

The examples shown illustrate several points. As already noted, the coupled QMR algorithm has a rather smooth and almost monotone convergence behavior. It makes available a residual norm upper bound that is a very good indicator of the convergence of the method. Finally, while it is possible to construct examples with arbitrary look-ahead structure, numerical experience seems to indicate that, on the average, the look-ahead strategy does not build many look-ahead blocks. Indeed, the vast majority of the steps taken by the coupled two-term look-ahead Lanczos process are regular steps; furthermore, from the look-ahead steps of size greater than 1 taken, the majority are blocks of size 2 .

\section{Concluding remarks}

We have presented details of an implementation of a new look-ahead algorithm for constructing Lanczos vectors based on coupled two-term recurrences instead of the usual three-term recurrences. We then discussed a new implementation of the quasi-minimal residual algorithm, using the coupled process to build the basis for the Krylov subspace. While the theoretical results derived for the original 


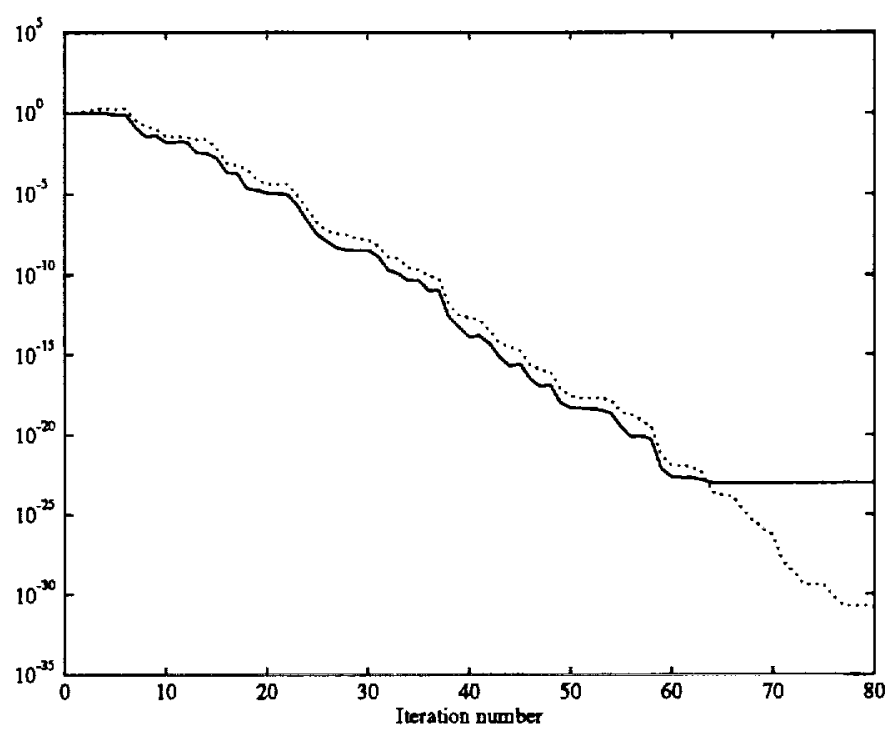

Figure 3: Convergence curves for Example 6.3.

algorithm carry over to the new one, the latter was shown in examples to have better numerical properties.

FORTRAN 77 codes for the coupled-two term look-ahead procedure and the resulting new implementation of the QMR algorithm can be obtained electronically from the authors (freund@research.att.com or na.nachtigal@na-net.ornl.gov). We note that FORTRAN 77 codes for the original implementation of QMR and the underlying look-ahead Lanczos algorithm are available from netlib by sending an email message consisting of the single line "send lalqmr from misc" to netlib@ornl.gov or netlib@research.att.com.

\section{Acknowledgements}

- We would like to thank V. Venkatakrishnan and W. McQuain for providing the matrices for Examples 6.2 and 6.3 .

\section{References}

[1] O. Axelsson, A survey of preconditioned iterative methods for linear systems of 
algebraic equations, BIT 25 (1985) 166-187.

[2] D. Baxter, J. Saltz, M. Schultz, S. Eisenstat, and K. Crowley, An experimental study of methods for parallel preconditioned Krylov methods, Technical Report RR-629, Department of Computer Science, Yale University, New Haven, Connecticut, 1988.

[3] R.W. Freund, M.H. Gutknecht, and N.M. Nachtigal, An implementation of the look-ahead Lanczos algorithm for non-Hermitian matrices, SIAM J. Sci. Comput. 14 (1993), to appear.

[4] R.W. Freund and M. Hochbruck, On the use of two QMR algorithms for solving singular systems and applications in Markov chain modeling, Journal of Numerical Linear Algebra with Applications 2 (1993), to appear.

[5] R.W. Freund and N.M. Nachtigal, QMR: a quasi-minimal residual method for nonHermitian linear systems, Numer. Math. 60 (1991), 315-339.

[6] R.W. Freund and N.M. Nachtigal, An implementation of the QMR method based on coupled two-term recurrences, Technical Report 92.15, RIACS, NASA Ames Research Center, Moffett Field, California, June 1992.

[7] C. Lanczos, An iteration method for the solution of the eigenvalue problem of linear differential and integral operators, J. Res. Nat. Bur. Standards 45 (1950), 255-282.

[8] W.D. McQuain, C.J. Ribbens, L.T. Watson, and R.C. Melville, Preconditioned iterative methods for sparse linear algebra problems arising in circuit simulation, Technical Report 92-07, Department of Computer Science, Virginia Polytechnic Institute and State University, Blacksburg, Virginia, March 1992.

[9] Y. Saad, ILUT: a dual threshold incomplete LU factorization, Research Report UMSI 92/38, University of Minnesota Supercomputer Institute, Minneapolis, Minnesota, March 1992.

[10] V. Venkatakrishnan and T.J. Barth, Unstructured grid solvers on the iPSC/860, Parallel Computational Fluid Dynamics, Rutgers, New Jersey, May 1992. 